\title{
Nucleon and N-Delta Electromagnetic Transition Form Factors
}

Franz Gross*, G. Ramalho 1 and M. T. Pena 1**

*College of William and Mary, Williamsburg, Virginia 23185, USA Thomas Jefferson National Accelerator Facility, Newport News, VA 23606, USA

1 Centro de Fisica Tedrica e de Particulas, Av. Rovisco Pais, 1049-001 Lisboa, Portugal

**Department of Physics, Instituto Superior Tecnico, Av. Rovisco Pais, 1049-001 Lisboa, Portugal

We consider the baryons as three constituent valence quark systems. Their dynamics is described by the covariant Spectator formalism [1,2] for a quark-diquark system, where the diquark is always on its massshell. The electromagnetic interaction is considered in the relativistic impulse interaction (RIA) where the

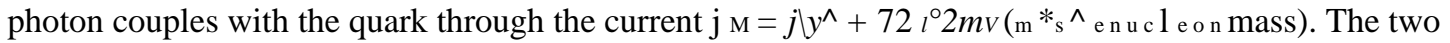
form factors j'l and 72 account for all QCD mechanisms ( $q q$ pairs, pion cloud and gluon sea effects). Only the 71 form factor includes pion cloud effects in its isovector part.

The nucleon wave function consists of spin-0 (isospin-0) and spin-1 (isospin-l) components written in terms of the diquark polarization vectors and the nucleon Dirac spinor [1]. Furthermore, it verifies the Dirac equation and generates the correct structure for its non-relativistic limit. Current conservation is also satisfied. The Jlab polarization data of the electromagnetic nucleon elastic form factors are described $[3,4]$ when one assumes an S-state for the quark-diquark system [1], which means that the data does not signal any angular dependence in the wave function. The results show that spherical charge and matter distributions are compatible with the data, even when we consider Spin Direction Dependent density definitions [5]. The explicit consideration of the pion cloud effects definitively improves the description of the nucleon form factors [1].

We also calculated the N-Delta transition form factors. Preliminary results considering the Delta wave function as a mixture of a S and a D state explain the magnetic dipole $G^{*}{ }_{M}$ and the electric quadrupole $G^{*} E$ data [6]. Improvements are underway in order to describe also the Coulomb quadrupole form factor GE.

[1] F. Gross, G. Ramalho and M. T. Pena, arXiv:nucl-th/0606029.

[2] F. Gross, Phys. Rev. 186, 1448 (1969); F. Gross and D. O. Riska, Phys. Rev. C36, 1928 (1987)

[3] V. Punjabi et al, Phys. Rev. C 71, 055202 (2005)

[4] New proton data analysis were supplied by J. Arrington, private communication.

[5] A. Kvinikhidze and G. A. Miller, Phys. Rev. C 73, 065203 (2006)

[6] V. V. Frolov et al, Phys. Rev. Lett. 82, 45 (1999); K. Joo et al. [CLAS Collaboration], Phys. Rev. Lett. 88, 122001 (2002); M. Ungaro, P. Staler, I. Aznauryan,

V. D. Burkert, K. Joo and L. C. Smith [CLAS Collaboration], arXiv:hep-ex/0606042. 